\title{
WELL-POSEDNESS OF THE DIFFERENCE SCHEMES OF THE HIGH ORDER OF ACCURACY FOR ELLIPTIC EQUATIONS
}

\author{
ALLABEREN ASHYRALYEV AND PAVEL E. SOBOLEVSKII
}

Received 22 May 2005; Accepted 14 August 2005

It is well known the differential equation $-u^{\prime \prime}(t)+A u(t)=f(t)(-\infty<t<\infty)$ in a general Banach space $E$ with the positive operator $A$ is ill-posed in the Banach space $C(E)=$ $C((-\infty, \infty), E)$ of the bounded continuous functions $\varphi(t)$ defined on the whole real line with norm $\|\varphi\|_{C(E)}=\sup _{-\infty<t<\infty}\|\varphi(t)\|_{E}$. In the present paper we consider the high order of accuracy two-step difference schemes generated by an exact difference scheme or by Taylor's decomposition on three points for the approximate solutions of this differential equation. The well-posedness of these difference schemes in the difference analogy of the smooth functions is obtained. The exact almost coercive inequality for solutions in $C(\tau, E)$ of these difference schemes is established.

Copyright (c) 2006 A. Ashyralyev and P. E. Sobolevskil. This is an open access article distributed under the Creative Commons Attribution License, which permits unrestricted use, distribution, and reproduction in any medium, provided the original work is properly cited.

\section{Introduction}

The role played by coercive inequalities in the study of local boundary-value problems for elliptic and parabolic differential equations is well known (see, e.g., $[10,11,16])$.

Coercivity inequalities in Hölder norms with a weight for the solutions of an abstract differential equation of elliptic type were established for the first time by Sobolevskiu [12]. Further in $[1,2,4,7,13]$ the coercive inequalities in Hölder norms with a weight were obtained for the solutions of various local and nonlocal boundary value problems for differential and difference equations of elliptic type. Finally in $[3,5,8,14]$ the corresponding coercivity inequalities in Hölder norms without a weight for the solutions of some local and nonlocal boundary value problems were obtained.

In the present paper we consider the differential equation

$$
-u^{\prime \prime}(t)+A u(t)=f(t) \quad(-\infty<t<\infty)
$$

in an arbitrary Banach space $E$ with positive operator $A$.

Hindawi Publishing Corporation

Discrete Dynamics in Nature and Society

Volume 2006, Article ID 75153, Pages 1-12

DOI 10.1155/DDNS/2006/75153 
A function $u(t)$ is called a solution of the problem (1.1) if the following conditions are satisfied:

(i) $u(t)$ is the bounded twice continuously differentiable on the real line $(-\infty, \infty)$;

(ii) the element $u(t)$ belongs to $D(A)$ for all $t \in(-\infty, \infty)$, and the function $A u(t)$ is bounded continuous for all $t \in(-\infty, \infty)$;

(iii) $u(t)$ satisfies (1.1).

A solution of the differential equation (1.1) defined in this manner will from now on referred to as a solution of the differential equation (1.1) in the space $C(E)=C((-\infty, \infty)$, $E$ ) of all bounded continuous functions $\varphi(t)$ defined on $(-\infty, \infty)$ with values in $E$ equipped with the norm

$$
\|\varphi\|_{C(E)}=\sup _{t \in(-\infty, \infty)}\|\varphi(t)\|_{E}
$$

The well-posedness in $C(E)$ of the differential equation (1.1) means that coercive inequality

$$
\left\|u^{\prime \prime}\right\|_{C(E)}+\|A u\|_{C(E)} \leq M\|f\|_{C(E)}
$$

is true for its solution $u(t) \in C(E)$ with some $M$, does not depend on $f(t) \in C(E)$. It is known that from the coercive inequality (1.3) it follows the positivity of the operator $A$ in the Banach space $E$, that means the operator $I \lambda+A$ has bounded in $E$ inverse $(I \lambda+A)^{-1}$ for any $\lambda \geq 0$, and estimate

$$
\left\|(\lambda I+A)^{-1}\right\|_{E \rightarrow E} \leq \frac{M}{1+\lambda}
$$

holds for some $1 \leq M<\infty$. It turns out that this positivity property of the operator $A$ in $E$ is necessary condition of well-posedness of the differential equation (1.1) in $C(E)$. The positivity of the operator $A$ in $E$ is not a sufficient condition for the well-posedness of the differential equation (1.1). As it turns out, problem (1.1) is not well-posed for all such operators. The counterexample given by Sobolevski1 [14].

It is known (see, e.g, $[9,12])$ that the operator $A^{1 / 2}$ has better spectral properties than the positive operator $A$. In particular, the operator $\lambda I+\sqrt{A}$ has a bounded inverse for any complex number $\lambda$ with $\operatorname{Re} \lambda \geq 0$, and the estimate

$$
\left\|(\lambda I+\sqrt{A})^{-1}\right\|_{E \rightarrow E} \leq M(|\lambda|+1)^{-1}
$$

is true for some $M \geq 1$. Thus, $\sqrt{A}$ is a strongly positive in $E$ operator, that is, the following estimates hold:

$$
\begin{gathered}
\left\|e^{-t \sqrt{A}}\right\|_{E \rightarrow E} \leq M e^{-\delta t}, \quad t\left\|\sqrt{A} e^{-t \sqrt{A}}\right\|_{E \rightarrow E} \leq M, \quad t>0, \delta>0, \\
\left\|(\sqrt{A})^{\beta}\left(e^{-t \sqrt{A}}-e^{-(t+\tau) \sqrt{A}}\right)\right\|_{E \rightarrow E} \leq \frac{M \tau^{\alpha}}{t^{\alpha}}, \quad 0 \leq \alpha, \beta \leq 1,0<t \leq t+\tau \leq 1 .
\end{gathered}
$$

That means if spectral angle $\varphi(A, E)$ of operator $A$ in $E$ equal $\pi$, then spectral angle $\varphi\left(A^{1 / 2}, E\right)$ of operator $A^{1 / 2}$ in $E$ less than $\pi / 2$. If the function $f(t)$ is not only continuous, but also bounded continuously differentiable, it is known that (see, e.g., [14]) the 
formula

$$
u(t)=\frac{1}{2} B^{-1} \int_{-\infty}^{\infty} e^{-|t-\tau| B} f(\tau) d \tau
$$

(Here and in this paper we will put $B=A^{1 / 2}$ ) gives a solution of differential equation (1.1) in $C(E)$.

In the papers $[8,14]$ the well-posedness of the differential equation (1.1) was established in the Banach space $C^{\alpha}(E)=C^{\alpha}((-\infty, \infty), E)(0<\alpha<1)$ obtained by completion of the set of $E$-valued smooth functions $\varphi(t)$ on $(-\infty, \infty)$ in the norm

$$
\|\varphi\|_{C^{\alpha}(E)}=\|\varphi\|_{C(E)}+\sup _{-\infty<t<t+\tau<\infty} \frac{\|\varphi(t+\tau)-\varphi(t)\|_{E}}{\tau^{\alpha}}
$$

and in the Banach space $C\left(E_{\alpha}\right)=C\left((-\infty, \infty), E_{\alpha}\right)$. Here, the Banach space $E_{\alpha}=E_{\alpha}\left(E, A^{1 / 2}\right)$ $(0<\alpha<1)$ consists of those $v \in E$ for which the norm (see, e.g, [6])

$$
\|v\|_{E_{\alpha}}=\sup _{z>0} z^{1-\alpha}\left\|A^{1 / 2} \exp \left\{-z A^{1 / 2}\right\} v\right\|_{E}+\|v\|_{E}
$$

is finite. Moreover, in the paper [14] the difference scheme second order of accuracy

$$
-\tau^{-2}\left(u_{k+1}-2 u_{k}+u_{k-1}\right)+A u_{k}=\varphi_{k}, \quad \varphi_{k}=f\left(t_{k}\right), \quad t_{k}=k \tau,-\infty<k<\infty
$$

for the approximate solutions of (1.1) was studied. The exact almost coercive inequality for solutions in $C(\tau, E)$ of this difference scheme was established.

In the present paper we consider two-step difference schemes of the high order of accuracy generated by an exact difference scheme or by Taylor's decomposition on three points for the approximate solutions of (1.1). The well-posedness of these difference schemes in the difference analogy of the smooth functions is obtained.The exact almost coercive inequality for solutions in $C(\tau, E)$ of these difference schemes is established.

\section{Difference schemes generated by an exact difference scheme}

The construction of two-step difference schemes of an arbitrary high order of accuracy for the approximate solutions of the differential equation (1.1) is based on the following theorem.

Theorem 2.1. Let $u\left(t_{k}\right)$ be a solution of the differential equation (1.1) at the grid points $t=t_{k}$. Then $\left\{u\left(t_{k}\right)\right\}_{-\infty}^{\infty}$ is the solution of the following second order difference equations:

$$
\begin{gathered}
-\tau^{-2}\left(u\left(t_{k+1}\right)-2 u\left(t_{k}\right)+u\left(t_{k-1}\right)\right)+\tau^{-2}(I-\exp \{-\tau B\})\left(u\left(t_{k+1}\right)+u\left(t_{k-1}\right)\right) \\
+\tau^{-2}(\exp \{-2 \tau B\}-I) u\left(t_{k}\right)=\psi_{k}, \\
\psi_{k}=(2 \tau B)^{-1}\left(\psi_{1, k}+\psi_{2, k+1}\right)-(2 \tau B)^{-1} \exp \{-\tau B\}\left(\psi_{1, k+1}+\psi_{2, k}\right), \\
\psi_{1, k}=\tau^{-1} \int_{t_{k-1}}^{t_{k}} \exp \left\{-\left(t_{k}-s\right) B\right\} f(s) d s, \\
\psi_{2, k}=\tau^{-1} \int_{t_{k-1}}^{t_{k}} \exp \left\{-\left(s-t_{k-1}\right) B\right\} f(s) d s, \quad t_{k}=k \tau, k \in(-\infty, \infty) .
\end{gathered}
$$


The proof of Theorem 2.1 is based on the formula (1.7) for the solution of the differential equation (1.1).

From (2.1) it is clear that for approximate solutions of the differential equation (1.1) it is necessary to approximate the expressions $\exp \{-\tau B\}$ and

$$
\tau^{-1} \int_{t_{k-1}}^{t_{k}} \exp \left\{-\left(t_{k}-s\right) B\right\} f(s) d s, \quad \tau^{-1} \int_{t_{k-1}}^{t_{k}} \exp \left\{-\left(s-t_{k-1}\right) B\right\} f(s) d s .
$$

Let us remark that in constructing difference schemes it is important to know how to construct a right-hand side $\psi_{k}^{l, j}$ that satisfies

$$
(2 \tau B)^{-1}\left(\psi_{1, k}+\psi_{2, k+1}\right)-(2 \tau B)^{-1} \exp \{-\tau B\}\left(\psi_{1, k+1}+\psi_{2, k}\right)-\psi_{k}^{l, j}=o\left(\tau^{l+j}\right),
$$

and is sufficiently simple. The choice formula $\psi_{k}^{l, j}$ is not unique. Let $f^{(\lambda)}(t) \in D\left(B^{m-\lambda}\right)$ for $0 \leq \lambda \leq m, 1 \leq m \leq l+j$. Using Taylor's formula, we obtain

$$
\begin{aligned}
\tau^{-1} \int_{t_{k-1}}^{t_{k}} \exp \left\{-\left(t_{k}-s\right) B\right\} f(s) d s & =\sum_{m=1}^{l+j} \sum_{\lambda=0}^{m}\left(\begin{array}{c}
m \\
\lambda
\end{array}\right) B^{m-\lambda} f^{(\lambda)}\left(t_{k}\right) \frac{(-1)^{m} \tau^{m}}{(m+1) !}+o\left(\tau^{l+j}\right), \\
\tau^{-1} \int_{t_{k-1}}^{t_{k}} \exp \left\{-\left(s-t_{k-1}\right) B\right\} f(s) d s & =\sum_{m=1}^{l+j} \sum_{\lambda=0}^{m}\left(\begin{array}{c}
m \\
\lambda
\end{array}\right)(-B)^{m-\lambda} f^{(\lambda)}\left(t_{k-1}\right) \frac{\tau^{m}}{(m+1) !}+o\left(\tau^{l+j}\right) .
\end{aligned}
$$

Applying the exact difference scheme (2.1) and formulas (2.4), we obtain $(l+j)$-order of accuracy two-step difference schemes

$$
\begin{gathered}
-\tau^{-2}\left(u_{k+1}-2 u_{k}+u_{k-1}\right)+\tau^{-2}\left(I-R_{j, l}(\tau B)\right)\left(u_{k+1}+u_{k-1}\right)+\tau^{-2}\left(R_{j, l}^{2}(\tau B)-I\right) u_{k}=\psi_{k}^{l, j}, \\
\psi_{k}^{l, j}=(2 \tau B)^{-1}\left(\psi_{1, k}^{l, j}+\psi_{2, k+1}^{l, j}\right)-(2 \tau B)^{-1} R_{j, l}(\tau B)\left(\psi_{1, k+1}^{l, j}+\psi_{2, k}^{l, j}\right), \\
\psi_{1, k}^{l, j}=\sum_{m=1}^{l+j} \sum_{\lambda=0}^{m}\left(\begin{array}{c}
m \\
\lambda
\end{array}\right) B^{m-\lambda} f^{(\lambda)}\left(t_{k}\right) \frac{(-1)^{m} \tau^{m}}{(m+1) !}, \\
\psi_{2, k}^{l, j}=\sum_{m=1}^{l+j} \sum_{\lambda=0}^{m}\left(\begin{array}{c}
m \\
\lambda
\end{array}\right)(-B)^{m-\lambda} f^{(\lambda)}\left(t_{k-1}\right) \frac{\tau^{m}}{(m+1) !}+o\left(\tau^{l+j}\right), \quad k \in(-\infty, \infty) .
\end{gathered}
$$

Here, the function $R_{j, l}(z)$ is constructed on the base of Padé's fractions

$$
R_{j, l}(z)=\frac{P_{j, l}(z)}{Q_{j, l}(z)}
$$

respectively

$$
P_{j, l}=1+a_{1} z+\cdots+a_{j} z^{j}, \quad Q_{j, l}(z)=1+b_{1} z+\cdots+b_{j} z^{l},
$$

where the coefficients $a_{i}, a_{i}=1, \ldots, j$, and $b_{i}, i=1, \ldots, l$, are uniquely defined from the 
condition

$$
\left|R_{j, l}(z)-e^{-z}\right|=o\left(|z|^{j+l+1}\right)
$$

for $|z| \rightarrow 0$.

Let $F(\tau, E)$ be the linear space of mesh functions $\varphi^{\tau}=\{\varphi k\}_{-\infty}^{\infty}$ with values in the Banach space $E$. Next on $F(\tau, E)$ we denote $C(\tau, E)$ and $C^{\alpha}(\tau, E)$ Banach spaces with the norms

$$
\begin{gathered}
\left\|\varphi^{\tau}\right\|_{C(\tau, E)}=\sup _{-\infty<k<\infty}\left\|\varphi_{k}\right\|_{E}, \\
\left\|\varphi^{\tau}\right\|_{C^{\alpha}(\tau, E)}=\left\|\varphi^{\tau}\right\|_{C(\tau, E)}+\sup _{-\infty<k<k+r<\infty} \frac{\left\|\varphi_{k+r}-\varphi_{k}\right\|_{E}}{(r \tau)^{\alpha}} .
\end{gathered}
$$

We say that the difference scheme $(2.5)$ is stable in $F(\tau, E)$ if we have the inequality

$$
\left\|\mathcal{u}^{\tau}\right\|_{F(\tau, E)} \leq M\left\|\psi_{j, l}^{\tau}\right\|_{F(\tau, E)},
$$

where $M$ is independent not only of $\psi_{j, l}^{\tau}$, but also on $\tau$.

We say that the difference scheme (2.5) is coercively stable (well-posed) in $F(\tau, E)$ if we have the coercive inequality

$$
\left\|\left\{\tau^{-2}\left(u_{k+1}-2 u_{k}+u_{k-1}\right)\right\}_{-\infty}^{\infty}\right\|_{F(\tau, E)} \leq M\left\|\psi_{j, l}^{\tau}\right\|_{F(\tau, E)},
$$

where $M$ is independent not only of $\psi_{j, l}^{\tau}$ but also of $\tau$.

It is known that if $A$ is a positive operator, then $A^{1 / 2}$ is a strongly positive operator. Since, difference schemes (2.5) are constructed by using an operator $A^{1 / 2}$, instead of $A$. Therefore, we have the following theorem.

Theorem 2.2. Let $j=l-1, l-2$. Then two-step difference schemes (2.5) are stable in $C^{\alpha}(\tau$, E), $0 \leq \alpha \leq 1$.

Since the differential equation (1.1) in the space $C(E)$ of bounded continuous functions defined on the real line with values in $E$ is not well-posed in the case of general positive operator $A$, then the well-posedness of the difference schemes $(2.5)$ in $C(\tau, E)$ norm does not take place uniformly with respect to $\tau>0$. This means that the coercive norm

$$
\begin{aligned}
\left\|u^{\tau}\right\|_{k(\tau, E)}= & \left\|\left\{\tau^{-2}\left(u_{k+1}-2 u_{k}+u_{k-1}\right)\right\}_{-\infty}^{\infty}\right\|_{C(\tau, E)} \\
& +\left\|\left\{\tau^{-2}\left(I-R_{j, l}(\tau B)\right)\left(u_{k+1}+u_{k-1}\right)+\tau^{-2}\left(R_{j, l}^{2}(\tau B)-I\right) u_{k}\right\}_{-\infty}^{\infty}\right\|_{C(\tau, E)}
\end{aligned}
$$

tends to $\infty$ as $\tau \rightarrow+0$. The investigation of difference schemes (2.5) permits us to establish the order of growth of this norm to $\infty$.

Theorem 2.3. Let $j=l-1, l-2$. Then for the solution of the two-step difference schemes (2.5) we have almost coercive inequality

$$
\left\|u^{\tau}\right\|_{k(\tau, E)} \leq M \min \left\{\ln \frac{1}{\tau}, 1+\left|\ln \|B\|_{E \rightarrow E}\right|\right\}\left\|\psi_{j, l}^{\tau}\right\|_{C(\tau, E)} .
$$


Note that if $B$ is the unbounded positive operator in $E$, then

$$
\min \left\{\ln \frac{1}{\tau}, 1+\left|\ln \|B\|_{E \rightarrow E}\right|\right\}=\ln \frac{1}{\tau} .
$$

Theorem 2.4. Let $j=l-1, l-2$. Then two-step difference schemes (2.5) are well-posed in $C^{\alpha}(\tau, E)$ and $C\left(\tau, E_{\alpha}\right), 0<\alpha<1$.

\section{Difference schemes generated by the Taylor's decomposition}

Let $f^{(2 i)}\left(t_{k}\right) \in D\left(A^{m-i}\right)$ for $0 \leq i \leq m, 0 \leq m \leq l-1$. We consider two-step difference schemes

$$
\begin{gathered}
-\tau^{-2}\left(u_{k+1}-2 u_{k}+u_{k-1}\right)+\sum_{m=0}^{l-1} \frac{2 \tau^{2 m}}{(2 m+2) !} A^{m+1} u_{k}=\varphi_{k}, \quad-\infty<k<\infty, \\
\varphi_{k}=\sum_{m=0}^{l-1} \frac{2 \tau^{2 m}}{(2 m+2) !} \sum_{i=0}^{m} A^{m-i} f^{(2 i)}\left(t_{k}\right), \quad t_{k}=k \tau,-\infty<k<\infty
\end{gathered}
$$

of $2 l$-order of accuracy of approximately solving (1.1). Difference schemes (3.1) generated by an exact difference scheme

$$
\begin{gathered}
-\tau^{-2}\left(v\left(t_{k+1}\right)-2 v\left(t_{k}\right)+v\left(t_{k-1}\right)\right)+\sum_{m=0}^{l-1} \frac{2 \tau^{2 m}}{(2 m+2) !} A^{m+1} v\left(t_{k}\right)=\psi_{k}, \\
\psi_{k}=\sum_{m=0}^{l-1} \frac{2 \tau^{2 m}}{(2 m+2) !} \sum_{i=0}^{m} A^{m-i} f^{(2 i)}\left(t_{k}\right)+\tau^{-2} \\
\times\left[\int_{t_{k-1}}^{t_{k}}\left(t-t_{k-1}\right) \int_{t_{k}}^{t} \frac{(t-s)^{2 l-1}}{(2 l-1) !} v^{(2 l+2)}(s) d s d t\right. \\
\left.+\int_{t_{k}}^{t_{k}+1}\left(t_{k+1}-t\right) \int_{t_{k}}^{t} \frac{(t-s)^{2 l-1}}{(2 l-1) !} v^{(2 l+2)}(s) d s d t\right] .
\end{gathered}
$$

This exact difference scheme generated by the Taylor's decomposition on three points.

This difference equation is uniquely solvable, and the following formula holds

$$
u_{k}=(I+\tau B)(2 I+\tau B)^{-1} B^{-1} \sum_{i=-\infty}^{\infty} R^{|k-i|}(\tau B) \varphi_{i} \tau,
$$

where

$$
B=B(\tau, A)=\frac{\tau^{2} A_{l}}{2}+\sqrt{\left(\frac{\tau^{2} A_{l}}{2}\right)^{2}+A_{l}}, \quad A_{l}=\sum_{m=0}^{l-1} \frac{2 \tau^{2 m}}{(2 m+2) !} A^{m+1} .
$$


Actually, the difference scheme (3.1) can be rewritten as the equivalent system of the first order linear difference equations

$$
\begin{gathered}
\tau^{-1}\left(u_{k}-u_{k-1}\right)+B u_{k}=w_{k}, \\
-\tau^{-1}\left(w_{k+1}-w_{k}\right)+B w_{k}=(I+\tau B) \varphi_{k},
\end{gathered}
$$

with operator $B=B(\tau, A)$. Note that $B(\tau, A) \neq A^{1 / 2}$ but then $B(\tau, A) \rightarrow A^{1 / 2}$ as $\tau \rightarrow 0$ and it has same spectral properties of $A^{1 / 2}$ under the strong positivity assumption for $A$. From the last system it follows the system of recursion formulas

$$
\begin{gathered}
u_{k}=R(\tau B) u_{k-1}+\tau R(\tau B) w_{k}, \\
w_{k}=R(\tau B) w_{k+1}+\varphi_{k},
\end{gathered}
$$

where $R(\tau B)=(I+\tau B)^{-1}$. Hence

$$
\begin{gathered}
u_{k}=\sum_{i=-\infty}^{k} R^{k-i+1}(\tau B) \tau w_{i} \\
w_{k}=\sum_{s=k}^{\infty} R^{s-k}(\tau B) \varphi_{s} \tau
\end{gathered}
$$

From that it follows that

$$
u_{k}=\sum_{i=-\infty}^{k} R^{k-i+1}(\tau B) \tau \sum_{s=i}^{\infty} R^{s-i}(\tau B) \varphi_{s} \tau .
$$

Since

$$
\begin{aligned}
& \sum_{i=-\infty}^{k} R^{k-i+1}(\tau B) \tau \sum_{s=i}^{\infty} R^{s-i}(\tau B) \varphi_{s} \tau \\
&=\sum_{s=-\infty}^{k} \tau \sum_{i=-\infty}^{s} R^{k+s+1-2 i}(\tau B) \varphi_{s} \tau+\sum_{s=k+1}^{\infty} \tau \sum_{i=-\infty}^{k} R^{k+s-2 i+1}(\tau B) \varphi_{s} \tau \\
&=\left(I-R^{2}(\tau B)\right)^{-1} \sum_{s=-\infty}^{k} \tau R^{k-s+1}(\tau B) \varphi_{s} \tau \\
& \quad+\left(I-R^{2}(\tau B)\right)^{-1} \sum_{s=k+1}^{\infty} \tau R^{s-k+1}(\tau B) \varphi_{s} \tau \\
&=\left(I-R^{2}(\tau B)\right)^{-1} \sum_{s=1}^{\infty} \tau\left(R^{|k-s|+1}(\tau B)-R^{k+s}(\tau B)\right) \varphi_{s} \tau
\end{aligned}
$$

we have that

$$
u_{k}=\left(I-R^{2}(\tau B)\right)^{-1} \sum_{s=-\infty}^{\infty} \tau R^{|k-s|+1}(\tau B) \varphi_{s} \tau .
$$

From this formula it follows the formula (3.3). 
As above, let us give the definition of the stability and coercively stable of the difference equation (3.1).

The difference equation (3.1) is said to be stable in $F_{\tau}(E)$ if we have the inequality

$$
\left\|u^{\tau}\right\|_{F(\tau, E)} \leq M\left\|\varphi^{\tau}\right\|_{F(\tau, E)},
$$

where $M$ is independent not only of $\varphi^{\tau}$ but also of $\tau$.

The difference equation (3.1) is said to be well-posed (coercively stable) in $F_{\tau}(E)$ if we have the coercive inequality

$$
\left\|\left\{\tau^{-2}\left(u_{k+1}-2 u_{k}+u_{k-1}\right)\right\}_{-\infty}^{\infty}\right\|_{F(\tau, E)}+\left\|\left\{\sum_{m=0}^{l-1} \frac{2 \tau^{2 m}}{(2 m+2) !} A^{m+1} u_{k}\right\}_{-\infty}^{\infty}\right\|_{F(\tau, E)} \leq M\left\|\varphi^{\tau}\right\|_{F(\tau, E)},
$$

where $M$ is independent not only of $\varphi^{\tau}$ but also of $\tau$.

From the formula (3.3) it follows that the investigation of the stability and wellposedness of difference schemes (3.1) relies in an essential manner on a number of properties of the powers of the operator $(I+\tau B)^{-1}$. We are not able to obtain the estimates for powers of the operator $(I+\tau B)^{-1}$ in the general cases of operator $A$. However, for the investigation of (3.1) it is necessary to construct an operator $B=B(\tau, A)$ and to give estimates

$$
\begin{aligned}
\left\|R^{k}(\tau B)\right\|_{E \rightarrow E} \leq M(1+\delta \tau)^{-k}, & k \geq 1 \\
\left\|k \tau B R^{k}(\tau B)\right\|_{E \rightarrow E} \leq M(1+\delta \tau)^{-k}, & k \geq 1 .
\end{aligned}
$$

The proof of estimates (3.13) is based upon three theorems.

Theorem 3.1 (a real criterion for strong positivity [13]). A necessary and sufficient condition for $B$ to be strongly positive is that the estimates (3.13) are satisfied.

Theorem 3.2 ([13]). If $A$ is a strongly positive operator, then the operator $B$ denoted by

$$
B=\frac{1}{2} \tau A+\sqrt{\frac{1}{4}\left(\tau^{2} A\right)^{2}+A}
$$

is a strongly positive operator.

THeORem 3.3 ([2]). If $A$ is a strongly positive operator with spectrum angle $\phi(A, E) \leq \pi / 2 l$ then the operator $A_{l}$ denoted by formula (3.1) is also a strongly positive operator.

We have the following results.

Theorem 3.4. Let $A$ is a strongly positive operator in a Banach space $E$ with spectral angle $\phi(A, E)<\pi / 2 l$. Then difference problem (3.1) is stable in $C^{\alpha}(\tau, E),(0 \leq \alpha \leq 1)$. For the solutions of the difference problem (3.1) satisfy the stability inequalities

$$
\left\|u^{\tau}\right\|_{C^{\alpha}(\tau, E)} \leq M\left\|\varphi^{\tau}\right\|_{C^{\alpha}(\tau, E)}
$$

where $M$ does not depend on $\varphi^{\tau}, \alpha$ and $\tau$. 
Theorem 3.5. Let $A$ is a strongly positive operator in a Banach space $E$ with spectral angle $\phi(A, E)<\pi / 2 l$. Then the solutions of the difference problem (3.1) in $C(\tau, E)$ obey the almost coercive inequality

$$
\begin{aligned}
& \left\|\left\{\tau^{-2}\left(u_{k+1}-2 u_{k}+u_{k-1}\right)\right\}_{-\infty}^{\infty}\right\|_{C(\tau, E)}+\left\|\left\{\sum_{m=0}^{l-1} \frac{2 \tau^{2 m}}{(2 m+2) !} A^{m+1} u_{k}\right\}_{-\infty}^{\infty}\right\|_{C(\tau, E)} \\
& \leq M_{1} \min \left\{\ln \frac{1}{\tau}, 1+\left|\ln \|B\|_{E \rightarrow E}\right|\right\}\left\|\varphi^{\tau}\right\|_{C(\tau, E)},
\end{aligned}
$$

where $M_{1}$ is independent not only of $\varphi^{\tau}$, but also of $\tau$.

Theorem 3.6. Let $A$ is a strongly positive operator in a Banach space E with spectral angle $\phi(A, E)<\pi / 2 l$. Then the solutions of the difference problem (3.1) in $C^{\alpha}(\tau, E)$ and $C\left(\tau, E_{\alpha}\right)$, $0<\alpha<1$ obey the coercive inequality

$$
\begin{aligned}
& \left\|\left\{\tau^{-2}\left(u_{k+1}-2 u_{k}+u_{k-1}\right)\right\}_{-\infty}^{\infty}\right\|_{C^{\alpha}(\tau, E)}+\left\|\left\{\sum_{m=0}^{l-1} \frac{2 \tau^{2 m}}{(2 m+2) !} A^{m+1} u_{k}\right\}_{-\infty}^{\infty}\right\|_{C^{\alpha}(\tau, E)} \\
& \leq \frac{M_{1}}{\alpha(1-\alpha)}\left\|\varphi^{\tau}\right\|_{C^{\alpha}(\tau, E)}, \\
& \left\|\left\{\tau^{-2}\left(u_{k+1}-2 u_{k}+u_{k-1}\right)\right\}_{-\infty}^{\infty}\right\|_{C\left(\tau, E_{\alpha}\right)}+\left\|\left\{\sum_{m=0}^{l-1} \frac{2 \tau^{2 m}}{(2 m+2) !} A^{m+1} u_{k}\right\}_{-\infty}^{\infty}\right\|_{C\left(\tau, E_{\alpha}\right)} \\
& \leq \frac{M_{1}}{\alpha(1-\alpha)}\left\|\varphi^{\tau}\right\|_{C\left(\tau, E_{\alpha}\right)},
\end{aligned}
$$

where $M_{1}$ is independent not only of $\varphi^{\tau}, \alpha$, but also of $\tau$.

It is clear that

$$
\left\|A A_{l}^{-1}\right\|_{E \rightarrow E} \leq M
$$

From this estimate and Theorems 3.5-3.6 it follows that the following theorem.

Theorem 3.7. Let $A$ is a strongly positive operator in a Banach space $E$ with spectral angle $\phi(A, E)<\pi / 2 l$. Then the solutions of the difference problem (3.1) in $L_{p, \tau}(E)$ obey the almost coercive inequality

$$
\begin{array}{r}
\left\|\left\{\tau^{-2}\left(u_{k+1}-2 u_{k}+u_{k-1}\right)\right\}_{-\infty}^{\infty}\right\|_{C(\tau, E)}+\left\|\left\{A u_{k}\right\}_{-\infty}^{\infty}\right\|_{C(\tau, E)} \\
\leq M_{1} \min \left\{\ln \frac{1}{\tau}, 1+\left|\ln \|B\|_{E \rightarrow E}\right|\right\}\left\|\varphi^{\tau}\right\|_{C(\tau, E)},
\end{array}
$$

where $M_{1}$ is independent not only of $\varphi^{\tau}$, but also of $\tau$.

THeOREM 3.8. Let $A$ is a strongly positive operator in a Banach space $E$ with spectral angle $\phi(A, E)<\pi / 2 l$. Then the solutions of the difference problem (3.1) in $C^{\alpha}(\tau, E)$ and $C\left(\tau, E_{\alpha}\right)$, 
$0<\alpha<1$ obey the coercive inequality

$$
\begin{gathered}
\left\|\left\{\tau^{-2}\left(u_{k+1}-2 u_{k}+u_{k-1}\right)\right\}_{-\infty}^{\infty}\right\|_{C^{\alpha}(\tau, E)}+\left\|\left\{A u_{k}\right\}_{-\infty}^{\infty}\right\|_{C^{\alpha}(\tau, E)} \leq \frac{M_{1}}{\alpha(1-\alpha)}\left\|\varphi^{\tau}\right\|_{C^{\alpha}(\tau, E)}, \\
\left\|\left\{\tau^{-2}\left(u_{k+1}-2 u_{k}+u_{k-1}\right)\right\}_{-\infty}^{\infty}\right\|_{C\left(\tau, E_{\alpha}\right)}+\left\|\left\{A u_{k}\right\}_{-\infty}^{\infty}\right\|_{C\left(\tau, E_{\alpha}\right)} \leq \frac{M_{1}}{\alpha(1-\alpha)}\left\|\varphi^{\tau}\right\|_{C\left(\tau, E_{\alpha}\right)},
\end{gathered}
$$

where $M_{1}$ is independent not only of $\varphi^{\tau}, \alpha$, but also of $\tau$.

\section{Application}

We consider $2 m$-order multidimensional elliptic equation

$$
-\frac{\partial^{2} u}{\partial y^{2}}+\sum_{|r|=2 m} a_{r}(x) \frac{\partial^{|\tau|} u}{\partial x_{1}^{r_{1}} \cdots \partial x_{n}^{r_{n}}}+\delta u(y, x)=f(y, x), \quad-\infty<y<\infty, x \in \mathbb{R}^{n}
$$

where $a_{r}(x)$ and $f(y, x)$ are given sufficiently smooth functions and $\alpha_{r}(x)>0, \delta>0$ is the sufficiently large number. Here $r \in \mathbb{R}^{n}$ is a vector with nonnegative integer components, $|r|=r_{1}+\cdots+r_{n}$.

Now, the abstract theorems given from above are applied in the investigation of difference schemes of higher order of accuracy with respect to the set all variables for approximate solution of the differential equation (4.1). The discretization of problem (4.1) is carried out in two steps. In the first step let us give the difference operator $A_{h}^{x}$ by the formula

$$
A_{h}^{x} u_{x}^{h}=\sum_{2 m \leq|r| \leq S} b_{r}^{x} D_{h}^{r} u_{x}^{h}+\delta u_{x}^{h}
$$

The coefficients are chosen in such a way that the operator $A_{h}^{x}$ approximates in a specified way the operator (see [2])

$$
\sum_{|r|=2 m} a_{r}(x) \frac{\partial^{|r|}}{\partial x_{1}^{r_{1}} \cdots \partial x_{n}^{r_{n}}}+\delta
$$

We will assume that for $\left|\xi_{k} h\right| \leq \pi$ the symbol $A(\xi h, h)$ of the operator $A_{h}^{x}-\delta$ satisfies the inequalities

$$
(-1)^{m} A^{x}(\xi h, h) \geq M_{1}|\xi|^{2 m}, \quad\left|\arg A^{x}(\xi h, h)\right| \leq \phi<\phi_{0} \leq \frac{\pi}{2 l} .
$$

With the help of $A_{h}^{x}$ we arrive at the infinite system of ordinary differential equations

$$
-\frac{d^{2} v^{h}(y, x)}{d y^{2}}+A_{h}^{x} v^{h}(y, x)=f^{h}(y, x), \quad-\infty<y<\infty, x \in \mathbb{R}_{h}^{n} .
$$


In the second step we replace differential equation (4.5) by the difference scheme

$$
\begin{gathered}
-\frac{1}{\tau^{2}}\left(u_{k+1}^{h}(x)-2 u_{k}^{h}(x)+u_{k-1}^{h}(x)\right)+\sum_{i=1}^{l} \alpha_{i}\left(A_{h}^{x}\right)^{i} \tau^{2 i-2} u_{k}^{h}(x)=\varphi_{k}^{h}(x), \\
\varphi_{k}^{h}(x)=\sum_{m=0}^{l-1} \frac{2 \tau^{2 m}}{(2 m+2) !} \sum_{l=0}^{m}\left(A_{h}^{x}\right)^{m-i} f^{h(2 i)}\left(y_{k}, x\right), \quad y_{k}=k \tau,-\infty<k<\infty, x \in R_{h}^{n} .
\end{gathered}
$$

Let us give a number of corollaries of the abstract theorems given in the above.

Theorem 4.1. Let $\tau$ and $h$ be a sufficiently small numbers. Then the solutions of the difference schemes (4.6) satisfy the following stability estimates:

$$
\left\|\left\{u_{k}^{h}\right\}_{-\infty}^{\infty}\right\|_{C^{\alpha}\left(\tau, C_{h}^{\beta}\right)} \leq M\left\|\left\{\varphi_{k}^{h}\right\}_{-\infty}^{\infty}\right\|_{C^{\alpha}\left(\tau C_{h}^{\beta}\right)}, \quad 0 \leq \alpha<1,0 \leq \beta<1,
$$

where $M$ does not depend on $\left\{\varphi_{k}^{h}\right\}_{-\infty}^{\infty}, \alpha, \beta, h$ and $\tau$.

The proof of Theorem 4.1 is based on the abstract Theorem 3.4 and the positivity of the operator $A_{h}^{x}$ in $C_{h}^{\beta}[6]$.

Theorem 4.2. Let $\tau$ and $h$ be a sufficiently small numbers. Then the solutions of the difference schemes (4.6) satisfy the following almost coercive stability estimates:

$$
\left\|\left\{\tau^{-2}\left(u_{k+1}^{h}-2 u_{k}^{h}+u_{k-1}^{h}\right)\right\}_{-\infty}^{\infty}\right\|_{C\left(\tau, C_{h}\right)} \leq M \ln \frac{1}{\tau+h}\left\|\left\{\varphi_{k}^{h}\right\}_{-\infty}^{\infty}\right\|_{C\left(\tau, C_{h}\right)},
$$

where $M$ does not depend on $\left\{\varphi_{k}^{h}\right\}_{-\infty}^{\infty}$, h and $\tau$.

The proof of Theorem 4.2 is based on the abstract Theorem 3.7, the positivity of the operator $A_{h}^{x}$ in $C_{h}$ and on the almost coercivity inequality for an elliptic operator $A_{h}^{x}$ in $C_{h}$ and on the estimate

$$
\min \left\{\ln \frac{1}{\tau}, 1+|\ln |\left|B_{h}^{x}\right|_{C_{h} \rightarrow C_{h}} \mid\right\} \leq M \ln \frac{1}{\tau+h} .
$$

Theorem 4.3. Let $\tau$ and $h$ be a sufficiently small numbers. Then the solutions of the difference schemes (4.6) satisfy the coercivity estimates:

$$
\begin{aligned}
& \left\|\left\{\tau^{-2}\left(u_{k+1}^{h}-2 u_{k}^{h}+u_{k-1}^{h}\right)\right\}_{-\infty}^{\infty}\right\|_{C^{\alpha}\left(\tau, C_{h}^{\beta}\right)} \\
& \quad \leq M(\alpha, \beta)\left\|\left\{\varphi_{k}^{h}\right\}_{-\infty}^{\infty}\right\|_{C^{\alpha}\left(\tau, C_{h}^{\beta}\right)}, \quad 0 \leq \alpha<1,0<\beta<1,
\end{aligned}
$$

where $M(\alpha, \beta)$ does not depend on $\left\{\varphi_{k}^{h}\right\}_{-\infty}^{\infty}$, h and $\tau$.

The proof of Theorem 4.3 is based on the abstract Theorem 3.8 and the positivity of the operator $A_{h}^{x}$ in $C_{h}^{\beta}$ and the coercivity inequality for an elliptic operator $A_{h}^{x}$ in $C_{h}^{\beta}$, $0<\beta<1$ and on the fact that for any $0<\beta<1 / 2 m$ the norms in the spaces $E_{\beta}\left(A_{h}^{x}, C_{h}\right)$ and $C_{h}^{2 m \beta}$ are equivalent uniformly in $h$ [6] and on the following theorem on the structure of the fractional spaces $E_{\alpha}\left(\left(A_{h}^{x}\right)^{1 / 2}, C_{h}\right)$. 
Theorem 4.4 [2]. Let $A$ is a strongly positive operator in a Banach space $E$ with spectral angle $\phi(A, E)<\pi / 2$. Then for $0<\alpha<1 / 2$ the norms of the spaces $E_{\alpha}\left(A^{1 / 2}, E\right)$ and $E_{\alpha / 2}(A, E)$ are equivalent.

\section{References}

[1] A. Ashyralyev, Coercive solvability of elliptic equations in spaces of smooth functions, Boundary Value Problems for Nonclassical Equations in Mathematical Physics (Russian) (Novosibirsk, 1989), no. 2, Akad. Nauk SSSR Sibirsk. Otdel. Inst. Mat., Novosibirsk, 1989, pp. 82-86.

[2] W Well-posed solvability of the boundary value problem for difference equations of elliptic type, Nonlinear Analysis. Theory, Methods \& Applications. An International Multidisciplinary Journal. Series A: Theory and Methods 24 (1995), no. 2, 251-256.

[3] __ On well-posedness of the nonlocal boundary value problems for elliptic equations, Numerical Functional Analysis and Optimization. An International Journal 24 (2003), no. 1-2, 1-15.

[4] A. Ashyralyev and K. Amanov, On coercive estimates in hölder norms, Izv. Akad. Nauk Turkmen. SSR Ser. Fiz.-Tekhn. Khim. Geol. Nauk 1 (1996), 3-10 (Russian).

[5] A. Ashyralyev and B. Kendirli, Well-posedness of the nonlocal boundary value problems for elliptic equations, Functional Differential Equations 9 (2002), no. 1-2, 33-55.

[6] A. Ashyralyev and P. E. Sobolevskiı̌, Well-Posedness of Parabolic Difference Equations, Operator Theory: Advances and Applications, vol. 69, Birkhäuser, Basel, 1994.

[7] L. M. Gershteyn and P. E. Sobolevskiŭ, Well-posedness of the a Banach space, Differentsial'nye Uravneniya 10(11) (1974), 2059-2061 (Russian).

[8] P. Grisvard, Elliptic Problems in Nonsmooth Domains, Monographs and Studies in Mathematics, vol. 24, Pitman, Massachusetts, 1985.

[9] S. G. Krein, Linear Differential Equations in a Banach Space, Nauka, Moscow, 1966.

[10] O. A. Ladyzhenskaya, V. A. Solonnikov, and N. N. Ural'tseva, Linear and Quasilinear Equations of Parabolic Type, Nauka, Moscow, 1967, English transl. Linear and Quasilinear Equations of Parabolic Type, Translations of Mathematical Monographs, 23, American Mathematical Society, Rhode Island, 1968.

[11] O. A. Ladyzhenskaya and N. N. Ural'tseva, Linear and Quasilinear Equations of Elliptic Type, Nauka, Moscow, 1973, English transl. of first edition: Linear and Quasilinear Elliptic Equations, Academic Press, New York, 1968.

[12] P. E. Sobolevskiŭ, Elliptic equations in a Banach space, Differencial'nye Uravnenija 4 (1968), 1346-1348 (Russian).

[13] The theory of semigroups and the stability of difference schemes, Operator Theory in Function Spaces (Proc. School, Novosibirsk, 1975) (Russian), Izdat. "Nauka" Sibirsk. Otdel., Novosibirsk, 1977, pp. 304-337, 344.

[14] W Well-posedness of difference elliptic equation, Discrete Dynamics in Nature and Society 1 (1997), no. 3, 219-231.

[15] H. Triebel, Interpolation Theory, Function Spaces, Differential Operators, North-Holland Mathematical Library, vol. 18, North-Holland, Amsterdam, 1978.

[16] M. I. Vishik, A. D. Myshkis, and O. A. Oleinik, Partial Differential Equations, Mathematics in USSR in the Last 40 Years, 1917-1957, vol. 1, Fizmatgiz, Moscow, 1959, pp. 563-599.

Allaberen Ashyralyev: Department of Mathematics, Fatih University, Istanbul, Turkey

E-mail address: aashyr@fatih.edu.tr

Pavel E. Sobolevskiǐ: Institute of Mathematics, Universidade Federal do Ceara, Brazil

Current address: Institute of Mathematics, Hebrew University, Jerusalem, Israel

E-mail address: pavels@math.huji.ac.il 


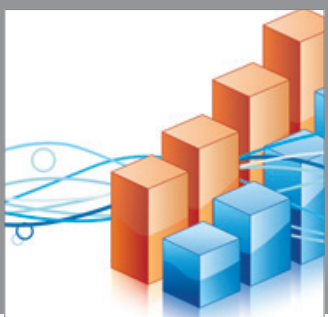

Advances in

Operations Research

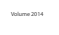

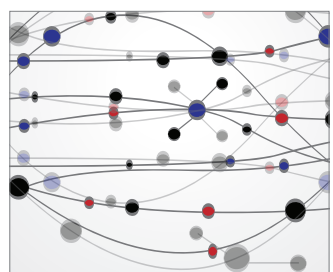

\section{The Scientific} World Journal
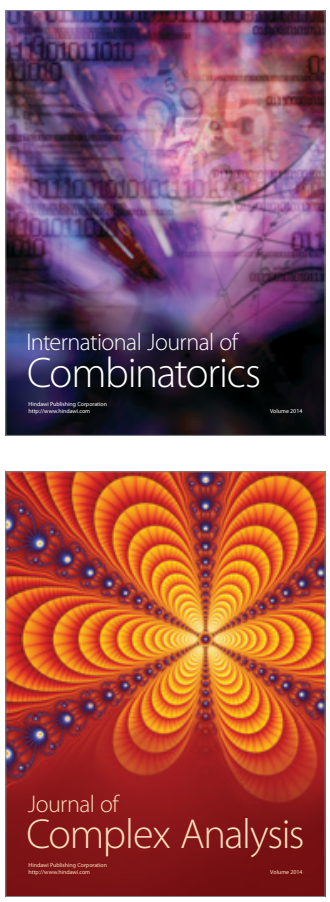

International Journal of

Mathematics and

Mathematical

Sciences
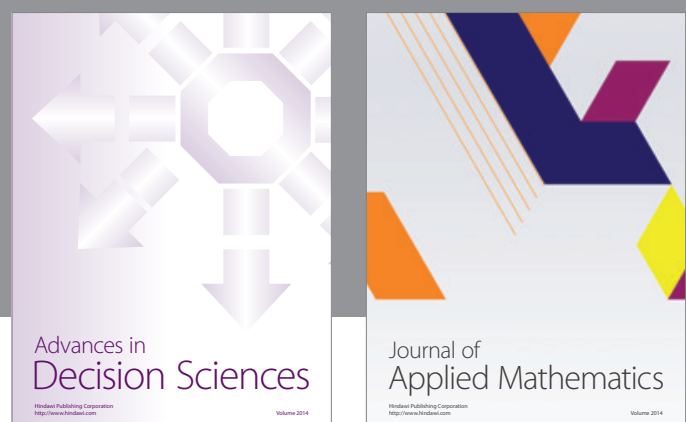

Journal of

Applied Mathematics
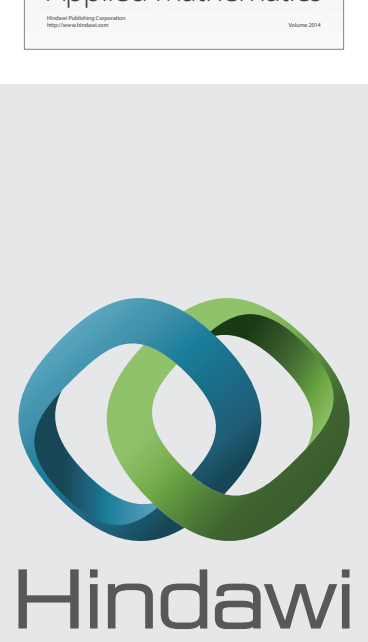

Submit your manuscripts at http://www.hindawi.com
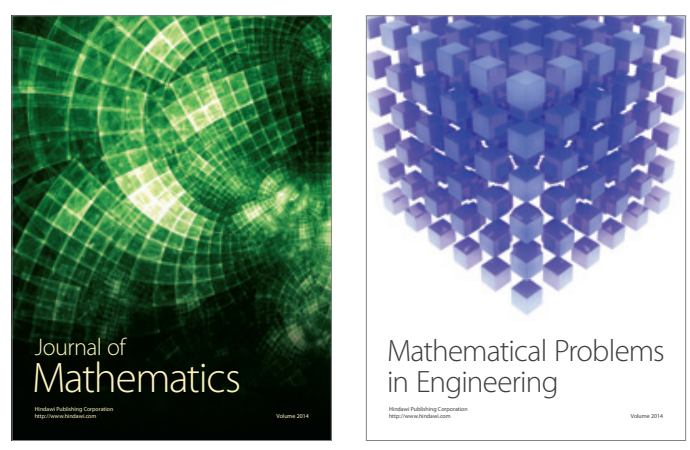

Mathematical Problems in Engineering
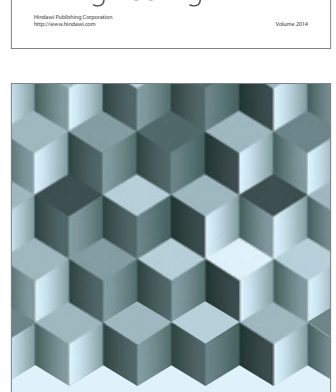

Journal of

Function Spaces
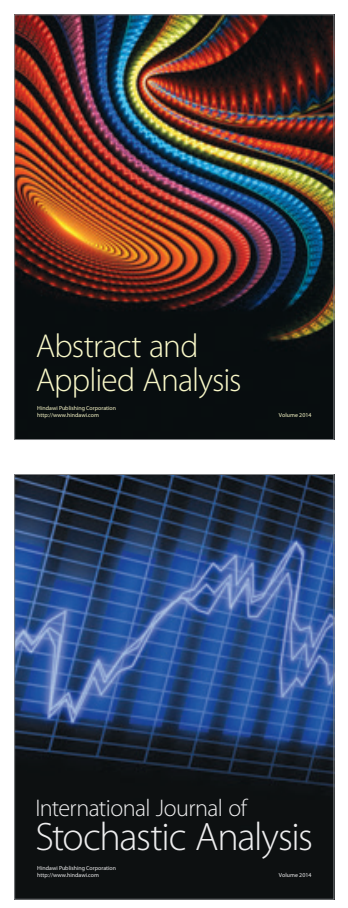

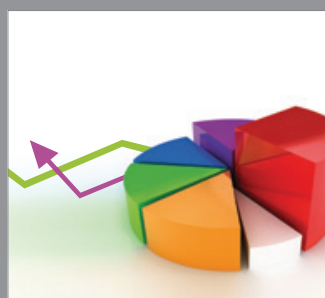

ournal of

Probability and Statistics

Promensencen
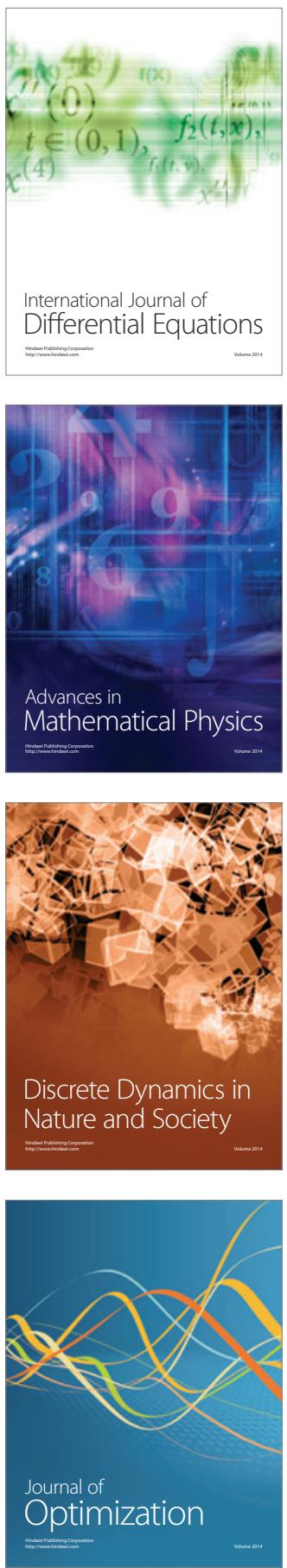